\title{
P03-1-51 Poster session
}

\section{Repeated social defeat induces social interaction deficits associated with the alteration of kynurenine pathway activity in the prefrontal cortex}

\author{
Kazuo Kunisawa ${ }^{1}$, Akihiro Mouri ${ }^{2,4}$, Aika Kosuge ${ }^{2}$, Tsubasa lida ${ }^{2}$, Yasuko Yamamoto ${ }^{3}$, \\ Kuniaki Saito ${ }^{3,4}$, Toshitaka Nabeshima ${ }^{1,4}$
}

${ }^{I}$ Advanced Diagnostic System Research Laboratory, Fujita Health University Graduate School of Health Science, Japan, ${ }^{2}$ Department of Regulatory Science for Evaluation \& Development of Pharmaceuticals \& Devices, Fujita Health University Graduate School of Health Science, Japan, ${ }^{3}$ Department of Disease Control and Prevention, Fujita Health

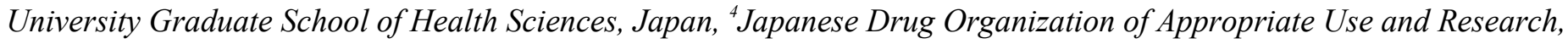
Japan

Chronic stress is associated with pathophysiology of psychiatric diseases such as major depressive disorder (MDD); however, the underlying mechanisms remain poorly understood. L-tryptophan (TRP) is metabolized via serotonin (5HT) and kynurenine (KYN) pathways. Previous studies have demonstrated that abnormality of both pathways is involved in the pathogenesis of MDD. In this study as depression animal model, adult C57BL/6J mice were exposed to chronic social defeat stress and measured contents of TRP, 5-HT, KYN and their metabolites in the prefrontal cortex. In the chronic social defeat stress, mice were exposed to aggressor ICR mouse for 10 consecutive days. Chronic stress reduced the duration of time spent at the interaction zone in the social interaction test. Chronic stress did not affect contents of 5HT and 5-hydroxyindole acetic acid in the prefrontal cortex. But, contents of TRP and KYN were significantly increased and decreased, respectively, in the prefrontal cortex of mice exposed to chronic stress. As a result, the ratio of KYN/TRP, represented the enzyme activity of indoleamine-2,3-dioxygenase (IDO), was decreased in the mice exposed to chronic stress. Double immunostaining showed the IDO1 immunoreactivity was mainly observed in neurons of the prefrontal cortex of control mice, and which was reduced in the mice exposed to chronic stress. These results suggest that the chronic social defeat stress induced depressive behavior and reduction of IDO1-mediated TRP metabolism in the prefrontal cortex. IDO1 may play crucial roles in the pathophysiology of MDD, and could be an attractive target for the antidepressants. 